\title{
Hipparchus' Eclipse Trios and Early Trigonometry
}

\author{
Dennis W. Duke \\ Department of Physics and School of Computational Science \\ Florida State University \\ USA
}

In 1973 Toomer suggested that the basic scale of ancient (ca. 500 AD) Indian sine tables, a circle of radius $R^{\prime}=3438$, was a remnant of even earlier Greek trigonometry, and that evidence for Greek use of that scale might be found in the otherwise curious ratios, $327^{2} / 3 / 3144$ and $2471 / 2 / 3122 \frac{1}{2}$, for the lunar anomaly that Ptolemy attributes to Hipparchus (Toomer 1973). Toomer was building upon an earlier suggestion by Neugebauer that ancient Indian sine tables compiled at angular intervals of $33 / 4^{\circ}$ might have been derived from early Greek chord tables compiled at angular intervals of $712^{\circ}$ (Neugebauer 1972), using the fundamental relation

$$
\sin \alpha=\frac{1}{2} \operatorname{crd} 2 \alpha \text {. }
$$

In Almagest 4.11 Ptolemy tells us that Hipparchus analyzed two trios of lunar eclipses in order to determine the size of the first lunar anomaly (Toomer 1984, pp. 211-216). Hipparchus first used an eccentric model to analyze a trio of eclipses observed in Babylon in about $300 \mathrm{BC}$ and found $e / R=3272 / 3 / 3144$. Hipparchus later used an epicycle model to analyze a trio of eclipses observed in Alexandria in about $200 \mathrm{BC}$ and found $r / R=2471 / 2 / 31221 / 2$. For reasons he did not explain, Toomer reversed Hipparchus' order and analyzed first the second trio, usually referred to as Trio B. The input data for an analysis of lunar eclipse trios are the time of each eclipse and longitude of the Moon at that time, and Ptolemy reports that the Hipparchan intervals for Trio B are:

$$
\begin{aligned}
& \text { B2 - B1: } 178^{\mathrm{d}} 6^{\mathrm{h}} \text { and } 180 ; 20^{\circ} \\
& \text { B3 - B2: } 176^{\mathrm{d}} 11^{\mathrm{h}} \text { and } 168 ; 33^{\circ}
\end{aligned}
$$

However, Toomer used instead a time interval B3 - B2 of $176^{\mathrm{d}} 1 / 3^{\mathrm{h}}$, i.e. one hour shorter than given above (Toomer 1984, p. 215, fn. 75). ${ }^{1}$ Using the shorter interval, Toomer found it necessary to attribute to Hipparchus a mistake in geometry in order to explain the Hipparchan answer 3271/2/3122 $1 / 2$ for Trio B. Using the longer (correct) interval, Toomer found that the ratio attributed to Hipparchus by Ptolemy was essentially correct, and so Hipparchus in fact did the geometry correctly, but the resulting numbers in the ratio no longer supported the hypothesis that Hipparchus was using a chord table based on a circle of radius $3438 .^{2}$ (Toomer did not give the resulting numbers, but they must have been close to the values 232 / 2924 found below). 
On the other hand, Hipparchus' intervals for the first trio, Trio A, are:

$$
\begin{aligned}
& \text { A2 - A1: } 177^{\mathrm{d}} 133^{\mathrm{h}} \mathrm{h}^{\mathrm{h}} \text { and } 173-1 / 8^{\circ} \\
& \text { A3 - A2: } 177^{\mathrm{d}} 1{ }^{\mathrm{h}} \mathrm{s}^{\mathrm{h}} \text { and } 175+1 / 8^{\circ}
\end{aligned}
$$

Toomer analyzed this trio more or less correctly, and found $e / R=338 / 3134$, clearly suggesting that (a) his hypothesis that Hipparchus had used a chord table of radius $R^{\prime}=3438$ was correct, and (b) getting the exact Hipparchan numbers 327 $/ 3$ and 3144 was most likely a matter of numerical rounding and minor miscalculation. As Ptolemy explains in Almagest 4.11, even small adjustments in the input intervals can result in large variation in the output answers.

In the following we repeat Toomer's analysis of Trio A and confirm the conclusion that Hipparchus was very likely using a base 3438 chord table. In addition, we find an alternate path through the computation of Trio B that explains the absolute numbers $2471 / 2$ and $3122 \frac{1}{2}$ and sheds light on another numerical convention that Hipparchus was apparently using.

\section{Preliminaries}

Before diving deep into a thicket of tedious numerical analysis, it will be useful to discuss some preliminary results that may help in establishing and maintaining a useful perspective. First, the few geometrical tools needed are summarized in the Appendix. The principal assumption of Neugebauer and Toomer is that Hipparchus was using a chord table with entries derived from a circle of circumference 21,600, the number of arc minutes in $360^{\circ}$. Then starting with a reasonably accurate value for $\pi$ and the values of $\operatorname{crd}\left(90^{\circ}\right)$ and $\operatorname{crd}\left(60^{\circ}\right)$, which amounts to having estimates of $\sqrt{2}$ and $\sqrt{3}$, one can use simple theorems $s^{3}$ that relate $\operatorname{crd}\left(180^{\circ}-\alpha\right)$ and $\operatorname{crd}(\alpha / 2)$ to $\operatorname{crd}(\alpha)$ to compute the chord entries in steps of $712^{\circ}$. One further assumes the use of simple linear interpolation to compute the chord of an arbitrary angle. The table in the appendix is a possible replica of Hipparchus' table.

Second, we note that using Hipparchus' intervals for Trio A and doing the trio analysis correctly, one finds

$$
\frac{e}{R}=\frac{6 ; 24,49}{60}=\frac{336.07}{3144}=\frac{3272 / 3}{3065.36} \simeq \frac{3272 / 3}{3144}=\frac{6 ; 15,11}{60}
$$

The $3^{\text {rd }}$ and $4^{\text {th }}$ ratios above show what the numerator and denominator would be in a correct calculation, if the denominator or numerator really was what Hipparchus gave. The final two terms are Hipparchus' result, and show that Hipparchus made a fairly significant error in the calculation, far larger than would occur through rounding. The corresponding relations for Trio B are

$$
\frac{r}{R}=\frac{4 ; 46,24}{60}=\frac{248.41}{3122 \frac{1}{2}}=\frac{247 \frac{1}{2}}{3111.03} \simeq \frac{247 \frac{1}{2}}{3122 \frac{1}{2}}=\frac{4 ; 46,30}{60}
$$


showing that rounding is likely adequate to account for the small discrepancy in this case. The important lesson for us is that starting from Hipparchus' intervals it is impossible to get to his final numbers without invoking some generally small but unknown and irrecoverable sequence of numerical rounding for both trios, plus some miscalculation for Trio A. Of course, the same would be true for the great majority of calculations in the Almagest if Ptolemy had not written out so many of the intermediate steps - an event unique in ancient sources.

Third, it is important to realize a point about distance scales in Hipparchus' (and Ptolemy's) trigonometry calculations. Since these were done without the aid of symbolic algebra as we know it, every length in a diagram has to be given a specific numerical value before it is used in calculation. In particular, the lengths of the distances $d=O B$ in Figures 1 and 2 in the Appendix are used early in the calculation, and must be assigned numerical values, so that we can eventually compute $R(d)$ for Trio A and $r(d)$ for Trio B as implicit functions of $d$. While we know from the derivations in the Almagest that Ptolemy always used $d=120$, his standard value for the hypotenuse of every reference triangle and the diameter of every reference circle, including his deferent circles, there is no reason to assume that Ptolemy's convention was used by Hipparchus. Indeed, we shall see below that Hipparchus appears to have used the value $d=3,162 \simeq 1,000 \sqrt{10}$, at least in Trio B. As further discussed below, it is likely that this value results from the use of the approximation $\pi \simeq \sqrt{10}$, which, like $R^{\prime}=3438$, is widely attested in Indian astronomy texts.

Finally, it might be helpful to anticipate how the numbers $3272 / 3,3144,2471 / 2$, and $3122 \frac{1}{2}$ appear at intermediate steps and survive in the final ratios. The strategy is to work through each calculation numerically, much as Ptolemy does throughout the Almagest, but to maintain all intermediate steps as ratios of integers with simple fractions, rather than reducing all intermediate numbers to sexagesimal fractions as in the Almagest. For trio A, one first computes what is effectively $R(d)$, followed by $R / e$ as a function of $R(d)$. For trio B, one first computes $r(d)$ and then $r / R$ as a function of $r(d)$. Naturally, though, at intermediate steps decisions must be made about clearing and simplifying fractions. As it turns out, the cleanest interpretation is that during the computations of Trio A, the number 3144 appears as the result of a chord calculation, and survives through to the end, thus enabling the recovery of the scale of the underlying chord table used, and at the same time rendering whatever value Hipparchus was using for $d$ irrecoverable. For Trio B, however, the opposite happens: the number $2471 / 2$ arises from combining the value of $d$ and a ratio of chord values at an intermediate step, and that number survives to the end. So in this case we can recover the value of $d$ being used, but the scale of the underlying chord table is lost when he divides the two chord values, the ratio being of course independent of the scale of the chord table.

\section{The Eccentric Model and Trio A}

We first summarize the geometrical solution of the lunar eclipse trio problem. To be sure there is no direct evidence that Hipparchus used this particular solution, and in principle there must be other variants. On the other hand, this is the solution Ptolemy gives in the Almagest, and using it does lead us to the known Hipparchan numbers. 


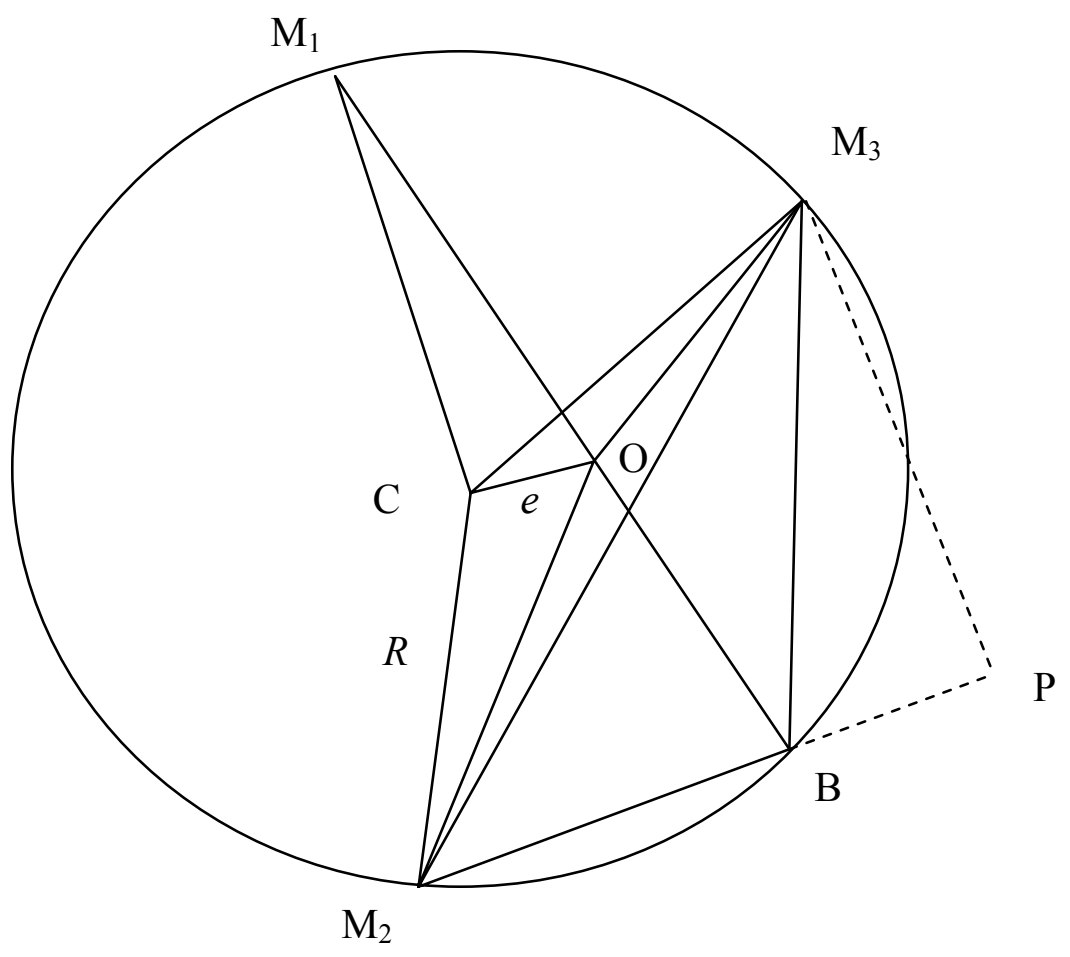

Figure 1. The lunar eccentric model

First we shall summarize the trigonometry solution in algebraic form. In Figure 1 the eclipses are at points $M_{1}, M_{2}, M_{3}$. The given angles are $M_{1} C M_{2}=\alpha_{1}, M_{2} C M_{3}=\alpha_{2}$, $M_{1} C M_{3}=\alpha_{3}$, and $M_{1} O M_{2}=\zeta_{1}, M_{2} O M_{3}=\zeta_{2}, M_{1} O M_{3}=\zeta_{3}$. We pick eclipse $M_{1}$ as a special reference eclipse, and extend the line $M_{l} O$ to intersect the circle at point $B$. Then for a fixed value of $d=O B$, which determines the intrinsic and theoretically arbitrary length scale in the figure, one finds in turn $M_{2} B, M_{3} B, M_{1} M_{3}, R, M_{1} B$, and $R / e$. The algorithm is:

1. Find the angles at point B: $M_{1} B M_{2}=\alpha_{1} / 2, M_{2} B M_{3}=180^{\circ}-\alpha_{2} / 2, M_{3} B M_{1}=\alpha_{3} / 2$.

2. Hence $O M_{2} B=\zeta_{1}-\alpha_{1} / 2$ and $O M_{3} B=\zeta_{3}-\alpha_{3} / 2$.

3. Sides of $M_{2} M_{3} B: \frac{M_{2} B}{d}=\frac{\operatorname{crd} 2\left(180^{\circ}-\zeta_{1}\right)}{\operatorname{crd} 2 O M_{2} B}, \frac{M_{3} B}{d}=\frac{\operatorname{crd} 2\left(180^{\circ}-\zeta_{3}\right)}{\operatorname{crd} 2 O M_{3} B}$,

$$
M_{3} P=M_{3} B \operatorname{crd} \alpha_{2}, M_{2} P=M_{2} B+M_{3} B \operatorname{crd}\left(180^{\circ}-\alpha_{2}\right) \text {, and }
$$$$
M_{2} M_{3}=\sqrt{M_{2} P^{2}+M_{3} P^{2}} \text {. }
$$

4. $R=\frac{M_{2} M_{3}}{\operatorname{crd} \alpha_{2}}$ (note that all lengths are proportional to $d$ ).

5. $M_{3} C B=\operatorname{crd}^{-1} \frac{M_{3} B}{R}$ and hence $M_{1} C B=M_{3} C B+\alpha_{3}$.

6. $M_{1} B=R \operatorname{crd} M_{l} C B$

7. $(R+e)(R-e)=R^{2}-e^{2}=O B \cdot M_{1} O=d\left(M_{1} B-d\right)$

8. $\frac{R}{e}=\frac{R / d}{\sqrt{(R / d)^{2}+1-M_{1} B / d}}$ 
To implement the calculation numerically, the first step is to convert the intervals in time and longitude into angles in anomaly and longitude. Thus we find $\alpha_{1}=\omega_{a}\left(t_{2}-t_{1}\right)$, $\alpha_{2}=\omega_{a}\left(t_{3}-t_{2}\right)$, and $\alpha_{3}=360^{\circ}-\alpha_{1}-\alpha_{2} ; \delta_{1}=\lambda_{2}-\lambda_{1}-\omega_{t}\left(t_{2}-t_{1}\right)$, $\delta_{2}=\lambda_{3}-\lambda_{2}-\omega_{t}\left(t_{3}-t_{2}\right)$, and $\delta_{3}=\delta_{1}-\delta_{2} ; \zeta_{i}=\alpha_{i}+\delta_{i}, i=1 . .3$. Computing these angles requires specific values for the lunar mean motion in anomaly and longitude, and the latter is conventionally computed from assumed values of the lunar synodic month and the length of the solar year. Ptolemy does not tell us at what stage in his career Hipparchus was doing these calculations, so it is marginally possible that he was using values other than those attributed to him in Almagest 4.2-3, possibly even sidereal values. We shall use the Almagest 4.2-3 values, but it remains the case that one source of minor variation in the answers is the assumed value of these parameters. Thus, using Hipparchus' Trio A intervals one finds by direct computation

$$
\begin{array}{ll}
\alpha_{1}=159 ; 59,14 & \zeta_{1}=153 ; 5,37 \\
\alpha_{2}=153 ; 24,33 & \zeta_{2}=155 ; 23,59 \\
\alpha_{3}=46 ; 36,11 & \zeta_{3}=51 ; 30,23
\end{array}
$$

Step by step, the solution is:

1. $M_{2} B=d \frac{\operatorname{crd} 2\left(180-\zeta_{1}\right)}{\operatorname{crd} 2\left(\zeta_{1}-\frac{\alpha_{1}}{2}\right)}=d \frac{3111 \frac{5}{8}}{6579}$

2. $M_{3} B=d \frac{\operatorname{crd} 2\left(180-\zeta_{3}\right)}{\operatorname{crd} 2\left(\zeta_{3}-\frac{\alpha_{3}}{2}\right)}=d \frac{5381 \frac{5}{7}}{3249 \frac{7}{9}}$

3. $M_{3} P=M_{3} B \frac{\operatorname{crd} \alpha_{2}}{2 R^{\prime}}=d \frac{5381 \frac{5}{7}}{3249 \frac{7}{9}} \cdot \frac{6991 \frac{5}{7}}{2 \cdot 3438}=d \frac{5540 \frac{4}{5}}{3438}$

4. $M_{2} P=M_{2} B+M_{3} B \frac{\operatorname{crd}\left(180-\alpha_{2}\right)}{2 R^{\prime}}=d \frac{3111 \frac{5}{8}}{6579}+d \frac{5381 \frac{5}{7}}{3249 \frac{7}{9}} \cdot \frac{1581 \frac{1}{4}}{2 \cdot 3438}=d \frac{2935 \frac{1}{3}}{3438}$

5. $M_{2} M_{3}=\sqrt{M_{2} P^{2}+M_{3} P^{2}}=d \frac{6270 \frac{1}{3}}{3438}$

6. $R=\frac{M_{2} M_{3}}{\operatorname{crd} \alpha_{2}}=d \frac{6270 \frac{1}{3}}{6691 \frac{5}{7}}=d \frac{3135 \frac{1}{7}}{3345 \frac{6}{7}}$ 
7. $\operatorname{crd} M_{3} C B=\frac{M_{3} B \cdot R^{\prime}}{R}=6076$

8. $M_{3} C B=124 ; 10^{\circ}$

9. $M_{1} C B=M_{3} C B+\alpha_{3}=170 ; 57^{\circ}$

10. $M_{1} B=R \frac{\operatorname{crd} M_{1} C B}{3438}=d \frac{6422 \frac{1}{7}}{3438}$

$11 \cdot \frac{R}{e}=\frac{R / d}{\sqrt{(R / d)^{2}+1-M_{1} B / d}}=\frac{3135 \frac{1}{7}}{\sqrt{\left(3135 \frac{1}{7}\right)^{2}+3345 \frac{6^{2}}{7}-3135 \frac{1}{7} \cdot 3345 \frac{6}{7} \cdot \frac{6854 \frac{1}{6}}{3438}}}=\frac{3135 \frac{1}{7}}{336}$

compared to Hipparchus' answer 3144 / 327 2/3. As mentioned earlier, Hipparchus made a mistake somewhere along the line and did not get the correct ratio.

In order to get Hipparchus' answer we have to invoke some amount of rounding and miscalculation, so the first step is to adjust something so that the correct numerical value for the ratio $R / e$ is produced. ${ }^{4}$ One simple way to accomplish this, out of an infinity of choices, is to assume that Hipparchus miscomputed $\zeta_{3}$ as 51;19,37, but did everything else precisely. Then he would get:

1. $M_{2} B=d \frac{\operatorname{crd} 2\left(180-\zeta_{1}\right)}{\operatorname{crd} 2\left(\zeta_{1}-\frac{\alpha_{1}}{2}\right)}=d \frac{3111 \frac{5}{6}}{6579}$

2. $M_{3} B=d \frac{\operatorname{crd} 2\left(180-\zeta_{3}\right)}{\operatorname{crd} 2\left(\zeta_{3}-\frac{\alpha_{3}}{2}\right)}=d \frac{5368 \frac{1}{9}}{3230 \frac{3}{4}}$

3. $M_{3} P=M_{3} B \frac{\operatorname{crd} \alpha_{2}}{2 R^{\prime}}=d \frac{5368 \frac{1}{9}}{3230 \frac{3}{4}} \cdot \frac{6991 \frac{4}{5}}{2 \cdot 3438}=d \frac{5559 \frac{1}{2}}{3438}$

4. $M_{2} P=M_{2} B+M_{3} B \frac{\operatorname{crd}\left(180-\alpha_{2}\right)}{2 R^{\prime}}=d \frac{3111 \frac{5}{6}}{6579}+d \frac{5368 \frac{1}{9}}{3230 \frac{3}{4}} \cdot \frac{1580 \frac{6}{7}}{2 \cdot 3438}=d \frac{2939 \frac{1}{2}}{3438}$

5. $M_{2} M_{3}=\sqrt{M_{2} P^{2}+M_{3} P^{2}}=d \frac{6288 \frac{3}{4}}{3438}$ 
6. $R=\frac{M_{2} M_{3}}{\operatorname{crd} \alpha_{2}}=d \frac{6288 \frac{3}{4}}{6691 \frac{4}{5}}=d \frac{3144 \frac{3}{8}}{3346}$

7. $\operatorname{crd} M_{3} C B=\frac{M_{3} B \cdot R^{\prime}}{R}=6078 \frac{5}{8}$

8. $M_{3} C B=124 ; 16^{\circ}$

9. $M_{1} C B=M_{3} C B+\alpha_{3}=170 ; 52^{\circ}$

10. $M_{1} B=R \frac{\operatorname{crd} M_{1} C B}{3438}=d \frac{6441 \frac{1}{3}}{3438}$

11. $\frac{R}{e}=\frac{R / d}{\sqrt{(R / d)^{2}+1-M_{1} B / d}}=\frac{3144 \frac{3}{8}}{\sqrt{\left(3144 \frac{3}{8}\right)^{2}+3346^{2}-3144 \frac{3}{8} \cdot 3346 \cdot \frac{6854 \frac{1}{6}}{3438}}}=\frac{3144 \frac{3}{8}}{327 \frac{5}{7}}$

In the above I have shown the numbers rounded to one digit fractions, but the computations are in fact done, in an Excel spreadsheet, to full precision at each step. Both of the final numbers are very close to the values Ptolemy attributes to Hipparchus. Thus, with or without accounting for the source of his errors, we can be certain that this is indeed the computational path that Hipparchus followed.

In order to show that this result is in no sense foreordained, we may use instead eclipse $M_{2}$ or $M_{3}$ as the reference, thus repeating the above calculation in two different ways. Then one finds that in order to get the crucial value 3144 in the numerator of the ratio for $R$, one must use the values $R^{\prime}=3589$ for $M_{2}$, and $R^{\prime}=3525$ for $M_{3}$, neither of which is a plausible, not to mention attested, value for the radius of a reference circle. The conclusion appears unavoidable and firm: the numbers 3144 and 3438 are unambiguously linked.

During the calculation above I have carried along the distance $d=O B$ symbolically, as did Toomer. It appears that whatever value Hipparchus might have used for $d$, he chose not to clear the fraction involving 3144 (other than canceling a factor of two) which shows up first in the computation of $M_{2} M_{3}$. We will see below that he apparently did clear this fraction when analyzing Trio B, thereby effectively masking the information on the chord table he was using. Thus it is entirely fortuitous that the evidence revealing the 3438 base of his chord table was revealed in the reported number 3144 of the Trio A ratio. 


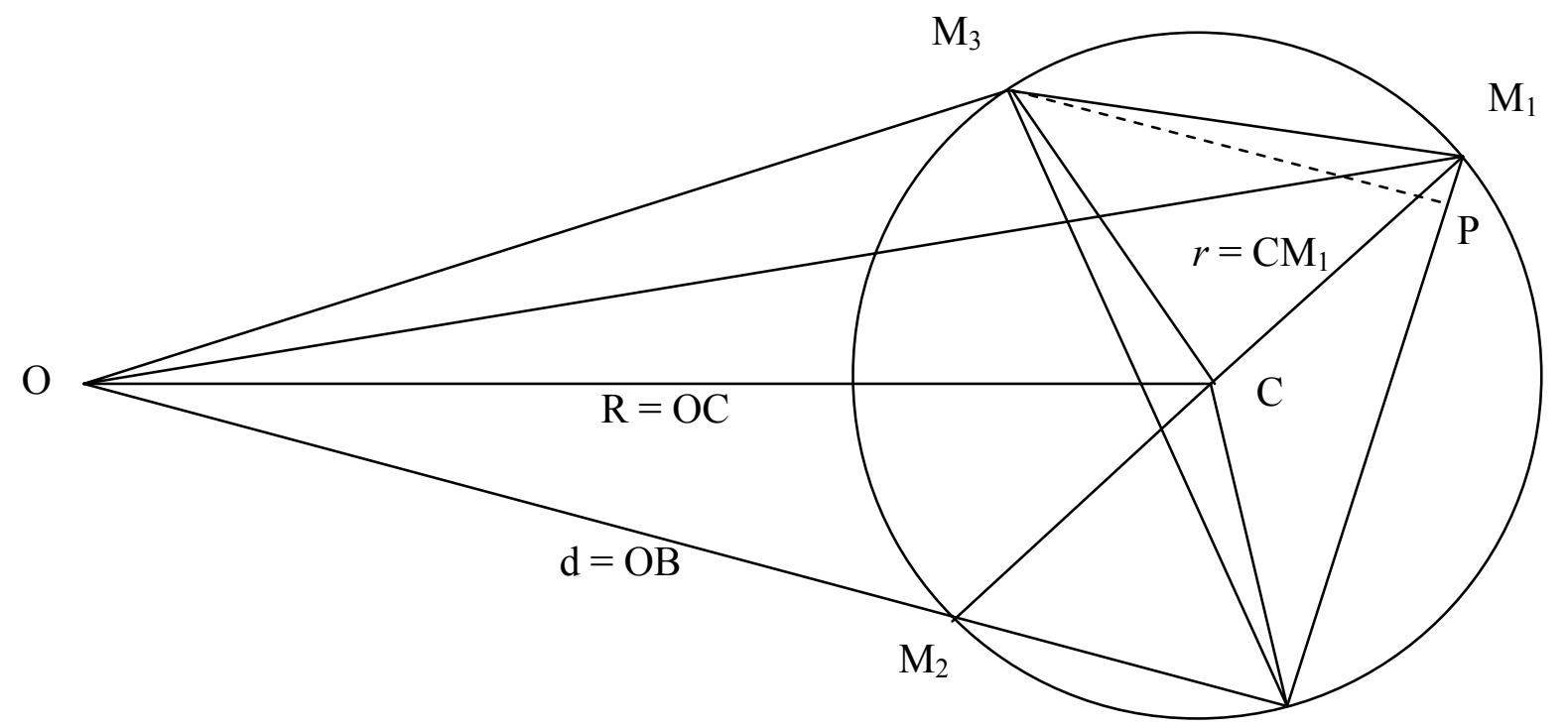

Figure 2. The lunar epicycle model

\section{The Epicycle Model and Trio B}

We now analyze the epicycle model for Trio B. In Figure 2 the eclipses are at points $M_{1}$, $M_{2}, M_{3}$ on a circle of radius $r$. The given angles are $M_{1} C M_{2}=\alpha_{1}, M_{2} C M_{3}=\alpha_{2}, M_{1} C M_{3}=$ $\alpha_{3}$, and $M_{1} O M_{2}=\delta_{1}, M_{2} O M_{3}=\delta_{2}, M_{1} O M_{3}=\delta_{3}$. Using eclipse $M_{2}$ as the reference eclipse, the solution strategy is very similar: for a fixed value of $d$, find $M_{1} B, M_{3} B, M_{1} M_{3}$, $r, M_{2} B$, and $r / R$. The analysis step by step is:

1. Find the angles at point B: $M_{1} B M_{3}=\alpha_{3} / 2, M_{2} B M_{3}=\alpha_{2} / 2, M_{1} B M_{2}=180^{\circ}-\alpha_{1} / 2$.

2. Hence $O M_{1} B=\alpha_{1} / 2-\delta_{1}$ and $O M_{3} B=180^{\circ}-\alpha_{2} / 2-\delta_{2}$.

3. Sides of $M_{1} M_{3} B: \frac{M_{1} B}{d}=\frac{\operatorname{crd} 2 \delta_{1}}{\operatorname{crd} 2 O M_{1} B}, \frac{M_{3} B}{d}=\frac{\operatorname{crd} 2 \delta_{2}}{\operatorname{crd} 2 O M_{3} B}, M_{3} P=M_{3} B \operatorname{crd} \alpha_{3}$,

$$
M_{1} P=M_{1} B-M_{3} B \operatorname{crd}\left(180^{\circ}-\alpha_{3}\right) .
$$

4. $M_{1} M_{3}=\sqrt{M_{1} P^{2}+M_{3} P^{2}}$

5. $r=\frac{M_{1} M_{3}}{\operatorname{crd} \alpha_{3}}$

6. $M_{3} C B=\operatorname{crd}^{-1} \frac{B M_{3}}{r}$ and hence $M_{2} C B=M_{3} C B-\alpha_{2}$.

7. $M_{2} B=r \operatorname{crd} M_{2} C B$

8. $R=\sqrt{d^{2}+r^{2}-d \cdot M_{2} B}$.

Using Hipparchus' Trio B intervals the angles $\alpha_{i}$ and $\delta_{i}$ are computed as in the eccentric model, and one finds 


$$
\begin{array}{ll}
\alpha_{1}=168 ; 50,0 & \delta_{1}=8 ; 21,24 \\
\alpha_{2}=140 ; 9,46 & \delta_{2}=8 ; 46,28 \\
\alpha_{3}=51 ; 0,13 & \delta_{3}=0 ; 25,4
\end{array}
$$

and, as mentioned earlier, a small discrepancy in the ratio $r / R$ compared to Hipparchus' answer $2471 / 2 / 3122 \frac{1}{2}$. In order to get Hipparchus' answer we again have to invoke a small amount of rounding and/or miscalculation. As in the eccentric case, the simplest route, of an infinity of choices, is to assume that the analyst miscomputed $\delta_{2}$ as $8 ; 44,8$ but did everything else precisely. Then he would get Hipparchus' numerical answer for the ratio $r / R$, no matter what the specific values for the numerator and denominator. However, following the same path as in Trio A using $R^{\prime}=3438$ does not give the specific values attributed to Hipparchus. Instead one finds $r=232$ and $R=2924$, or conversely, a circle radius of $R^{\prime}=3672$ is required to get the Hipparchan values $2471 / 2$ and $3122 \frac{1}{2}$. Using eclipses $M_{1}$ or $M_{3}$ as reference points, one finds that the required values of $R^{\prime}$ are instead 3178 and 3213 .

None of these values is particularly suggestive as the radius of a circle for a chord table, and so we must consider the possibility that Hipparchus followed a different path in this computation. Some variation in computational details by Hipparchus would not be unlikely in any case, given that (a) the models were different, and (b) the analyses were evidently made at different times, and possibly very different times. ${ }^{5}$ Now as discussed above, in the eccentric analysis of Trio A the specific value chosen for $d$ played no role. However, suppose that in the analysis of Trio B at step 5 Hipparchus used the specific value $d=3,162 \simeq 1,000 \sqrt{10}$ and, unlike his procedure in Trio A, combined all fractions, getting

$$
r=\frac{M_{1} M_{3}}{\operatorname{crd} \alpha_{3}}=3162 \frac{231 \frac{3}{4}}{2960 \frac{2}{5}} \simeq 247 \frac{1}{2} .
$$

Then in step 8 he would have computed

$$
\begin{aligned}
R & =\sqrt{d^{2}+r^{2}-d \cdot M_{2} B} \\
& =\sqrt{3162^{2}+2471^{2}-3162 \cdot 1061 / 7} \\
& \simeq 31221 / 2
\end{aligned}
$$

On the other hand, using eclipses $M_{1}$ or $M_{3}$ as reference points one finds that the required values of $d$ are instead 2988 and 3197, neither particularly suggestive as a reference circle diameter. Thus we may conclude that the numbers $2471 / 2$ and 3162 are firmly linked, and reveal Hipparchus' computational procedure for Trio B. ${ }^{6}$ Note that when Hipparchus combined the fractions in step 5 he effectively masked the radius of the chord circle that he was using. Thus, while it is certainly possible that he used $d=3162$ and $R^{\prime}=3438$ in both of the analyses, he might also have used 3438 for both values in Trio A, and 3162 for both values in Trio B. 
The rationale for the value of $d$ is in fact similar to that for $R^{\prime}=3438$. In numerous ancient Indian astronomy texts a common approximation for $\pi$ was $\sqrt{10}$, usually in the context of computing the diameter of a planet's orbit given the orbit's circumference (Pingree 1978, p. 557). Some of these sources also used the more accurate value 3.1416 for computing the equation of center and similar details in the planetary models. Now the rationale for the value $R^{\prime}=3438$ is that it is the radius of a circle whose circumference is $60^{\prime \prime \circ} \cdot 360^{\circ}=21,600^{\prime}$. It would not be unreasonable, then, to find someone using a convention in which a circle circumference is 10,000 , and assuming $\pi=\sqrt{10}$, the corresponding diameter is $d=1,000 \sqrt{10} \simeq 3,162$. Indeed, during the early days of trigonometry we probably should expect several coexisting conventions, until one eventually becomes dominant. We at least know from the Almagest that $d=120$ was a firmly established convention at Ptolemy's time. 
Appendix

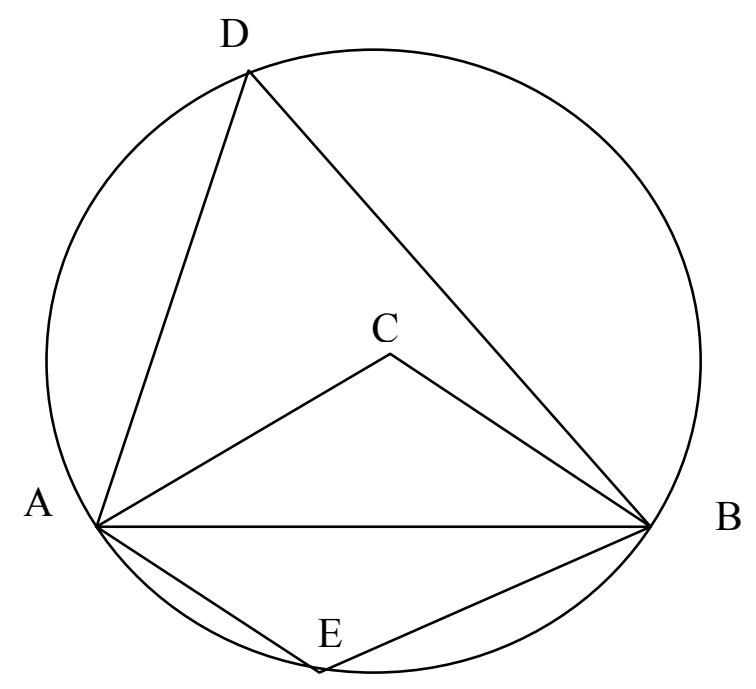

Consider the circle with center at $C$ and radius $C A=C B=R^{\prime}$. Then if angle $A C B=\alpha$, $\operatorname{crd} \alpha=A B / R^{\prime}=2 \sin \alpha / 2$

For any point $D$ on the longer arc, angle $A D B=\alpha / 2$, and for any point $E$ on the shorter arc, angle $A E B=180^{\circ}-\alpha / 2$.

Law of sines

$$
\frac{\operatorname{crd} 2 A}{a}=\frac{\operatorname{crd} 2 B}{b}=\frac{\operatorname{crd} 2 C}{c}
$$

where $a, b$ and $c$ are the sides of a triangle, and $A, B$ and $C$ are the opposite angles.
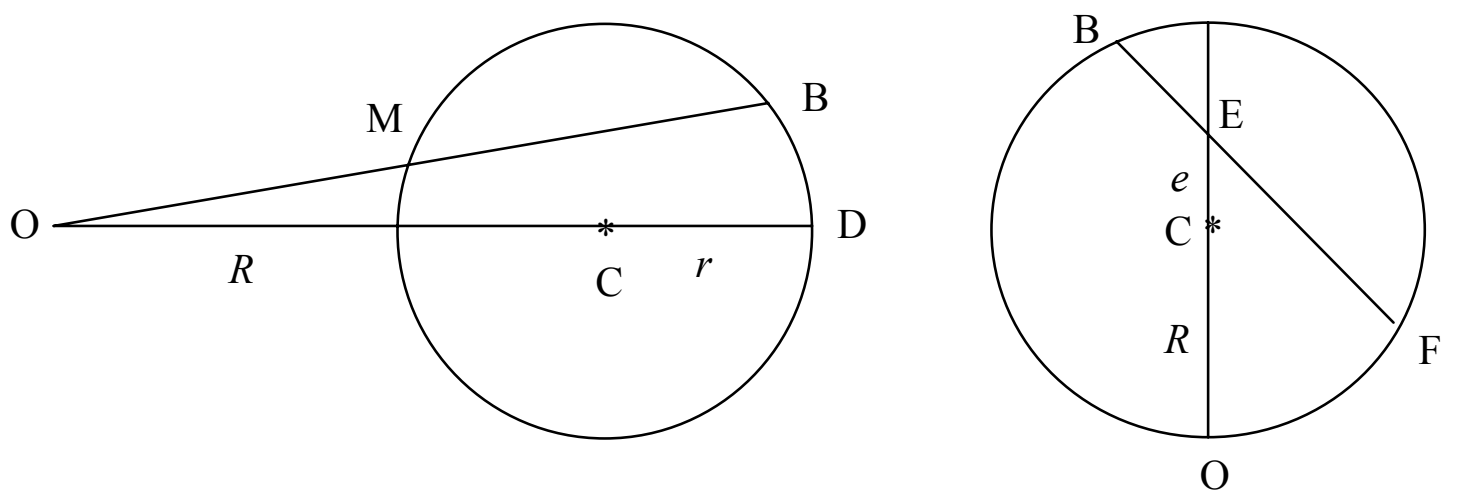

In the left figure, given $O C=R$ and $C D=r$, then $(R+r)(R-r)=R^{2}-r^{2}=O B \cdot O M$.

In the right figure, given $O C=R$ and $C E=e$, then $(R+e)(R-e)=R^{2}-e^{2}=F E \cdot E B$.

Assuming that Hipparchus used adequately accurate estimates of $\pi, \sqrt{2}$, and $\sqrt{3}$, the resulting chord table would be something close to the following. 
Table of chords

\begin{tabular}{||c|c||}
\hline Angle(degrees) & Chord \\
\hline 0 & 0 \\
\hline $71 / 2$ & 450 \\
\hline 15 & 897 \\
\hline $221 / 2$ & 1341 \\
\hline 30 & 1780 \\
\hline $371 / 2$ & 2210 \\
\hline 45 & 2631 \\
\hline $521 / 2$ & 3041 \\
\hline 60 & 3438 \\
\hline $671 / 2$ & 3820 \\
\hline 75 & 4186 \\
\hline $821 / 2$ & 4533 \\
\hline 90 & 4862 \\
\hline $971 / 2$ & 5169 \\
\hline 105 & 5455 \\
\hline $1121 / 2$ & 5717 \\
\hline 120 & 5954 \\
\hline $1271 / 2$ & 6166 \\
\hline 135 & 6352 \\
\hline $1421 / 2$ & 6511 \\
\hline 150 & 6641 \\
\hline $1571 / 2$ & 6743 \\
\hline 165 & 6817 \\
\hline $1721 / 2$ & 6861 \\
\hline 180 & 6875 \\
\hline & \\
\hline
\end{tabular}




\section{BIBLIOGRAPHY}

Heath T. L.

1897: The Works of Archimedes, Cambridge

Pingree, D.

1978: "History of Mathematical Astronomy in India", Dictionary of Scientific Biography, 15 , pp. 533-633.

Neugebauer, O.

1972: "On Some Aspects of Early Greek Astronomy", Proceedings of the American

Philosophical Society, 116, pp. 243-251.

Toomer, G. J.

1973: "The Chord Table of Hipparchus and the Early History of Greek Trigonometry", Centaurus 18, pp. 6-28.

Toomer, G. J.

1984: Ptolemy's Almagest, London 


\section{NOTES}

${ }^{1}$ Toomer explains that Heiberg's text in fact gives Hipparchus' time interval correctly, but a following sentence in the text, meant to give the differences in Ptolemy's and Hipparchus' intervals, is garbled. Manitius resolved the discrepancy incorrectly, and Toomer followed him.

${ }^{2}$ More precisely, chord tables are in fact based on a circle of a given diameter, and for a circle of circumference 21,600 the diameter $D^{\prime}$ is, to the nearest integer, 6875 , while the radius $R^{\prime}$ is, again to the nearest integer, 3438. The apparent discrepancy, that $D^{\prime} \neq 2 R^{\prime}$, is simply an accident of rounding.

${ }^{3}$ These were presumably well-known by Hipparchus' time, since the theorems are used explicitly by Archimedes in his "Measurement of the Circle", in which he inscribes and circumscribes a circle with 96-sided polygons to derive the bound $3 \frac{10}{71}<\pi<3 \frac{1}{7}$.

Archimedes begins with a hexagon and an estimate for $\sqrt{3}$ and by successive halving implicitly computes about two-thirds of the entries needed to populate a chord table with $71^{\circ}$ spacing. It would be straightforward to generate the remaining entries by starting with a square and an estimate of $\sqrt{2}$. For the details, see (Heath 1897).

${ }^{4}$ At this point, and again in Trio $\mathrm{B}$, it is not really necessary to change just one angle, and there is no intention to suggest that is what Hipparchus did. One could instead arrange a whole series of rounding errors and miscalculations in the following sequence of arithmetical operations. Either way, such fine details of the actual path followed by Hipparchus are irrecoverable, and, for our purposes, inconsequential.

${ }^{5}$ Ptolemy gives us no information about why Hipparchus was analyzing multiple eclipse trios, or what he concluded from his analyses. One reason to think that Hipparchus' analyses were done at substantially different times is that while the given intervals in time and longitude can be used to determine the parameters of the lunar model, the same intervals permit the recovery of the underlying solar model. In fact, assuming a standard Hipparchan solar model with only one inequality, and assuming that there are no errors in the intervals, a questionable assumption in itself, one finds the solar model parameters $e=7 ; 48$ and $A=76 ; 25^{\circ}$ for Trio A, and $e=3 ; 11$ and $A=46 ; 9^{\circ}$ for Trio B, both substantially different from the parameters Ptolemy attributes to Hipparchus in Almagest $3.1, e=2 ; 30$ and $A=65 ; 30^{\circ}$. While it is, of course, extremely unlikely that Hipparchus was actually using those models to compute the eclipse longitudes, and was instead using some alternate procedure that we do not now know, it hardly seems likely that he would be using such widely disparate solar models at the same time. Still, without more understanding of Hipparchus' scientific motivations, it is impossible to be certain about details such as the temporal sequence of his analyses.

${ }^{6}$ When Toomer recomputed Trio B with the (textually) correct intervals he found the ratio very nearly equal to $2471 / 2 / 3112 \frac{1}{2}$, rather than $2471 / 2 / 3122 \frac{1}{2}$ as given in the Almagest (see equation (2) above for the exact answers). He also realized that the former ratio is in slightly better agreement with what Ptolemy says is the ratio in his conventional base 60 norm, 4;46 / 60, and also with what Ptolemy says is the implied maximum equation of center, $4 ; 34^{\circ}$. Since all manuscripts are unanimous for $3122 \frac{1}{2}$, we can only conclude that the error, if there is one, was introduced at some point prior to the 
date of the earliest manuscript, about 800 A.D., and perhaps even before the time of Ptolemy. 